Artigo Original

Original Article

Carolina Finetti Marculino ${ }^{1}$

Camila Maia Rabelo ${ }^{2}$

Eliane Schochat ${ }^{2}$

Descritores

Testes auditivos/métodos

Ruído

Percepção auditiva

Audição

Criança

Keywords

Hearing tests/methods

Noise

Auditory perception

Hearing

Child

Endereço para correspondência:

Eliane Schochat.

R. Cipotânea, 51, Cidade Universitária, São Paulo (SP), Brasil, CEP: 05360-160.

E-mail: eschocha@usp.br

Recebido em: 19/1/2011

Aceito em: 1/9/2011

\section{0 teste Gaps-in-Noise: limiares de detecção de gap em crianças de 9 anos com audição normal}

\author{
Gaps-in-Noise test: gap detection thresholds in 9-year-old \\ normal-hearing children
}

\title{
RESUMO
}

Objetivo: Estabelecer os critérios de normalidade para o teste Gaps-in-Noise (GIN) em crianças de 9 anos de idade com audição normal, obter as médias dos limiares de detecção de intervalos de silêncio no ruído ( gap ) e verificar a influência das variáveis referentes a gênero e orelha. Métodos: Foram avaliados 40 indivíduos, 20 do gênero masculino e 20 do feminino, com idade entre 9 anos e 9 anos e 11 meses, normo-ouvintes. Os procedimentos realizados foram: anamnese, avaliação audiométrica, imitanciometria (timpanometria e pesquisa dos reflexos acústicos), teste Dicótico de Dígitos (aplicado para determinar a inclusão no estudo) e teste GIN. Os dados obtidos foram analisados estatisticamente. Resultados: Em relação ao desempenho no teste GIN por orelha, o resultado entre as orelhas direita e esquerda foi semelhante na população estudada. Em relação ao gênero, também não houve diferença. Nos indivíduos avaliados, a média dos limiares de detecção de intervalos de silêncio no ruído (gap) foi de 4,4 ms para orelha direita e 4,2 ms para orelha esquerda. Conclusão: Para crianças de 9 anos, os valores obtidos para a orelha direita e esquerda acrescidos de um desvio padrão podem ser usados como critério de normalidade para a idade independente da orelha ou gênero avaliado.

\begin{abstract}
Purpose: To establish the standard criteria for the Gaps-in-Noise (GIN) test in 9-year-old normal-hearing children; to obtain the mean gap detection thresholds; and to verify the influence of the variables gender and ear on the gap detection thresholds. Methods: Forty normal-hearing individuals, 20 male and 20 female, with ages ranging from 9 years to 9 years and 11 months, were evaluated. The procedures performed were: anamnesis, audiological evaluation, acoustic immittance measures (tympanometry and acoustic reflex), Dichotic Digits Test, and GIN test. The results obtained were statistically analyzed. Results: The results revealed similar performance of right and left ears in the population studied. There was also no difference regarding the variable gender. In the subjects evaluated, the mean gap detection thresholds were $4.4 \mathrm{~ms}$ for the right ear, and $4.2 \mathrm{~ms}$ for the left ear. Conclusion: The values obtained for right and left ear, as well as their standard deviations, can be used as standard criteria for 9-year-old children, regardless of ear or gender.
\end{abstract}

Trabalho realizado no Curso de Fonoaudiologia, Faculdade de Medicina, Universidade de São Paulo - USP São Paulo (SP), Brasil.

(1) Curso de Fonoaudiologia, Faculdade de Medicina, Universidade de São Paulo - USP - São Paulo (SP), Brasil. (2) Departamento de Fisioterapia, Fonoaudiologia e Terapia Ocupacional, Faculdade de Medicina, Universidade de São Paulo - USP - São Paulo (SP), Brasil. 


\section{INTRODUÇÃO}

De forma geral, o processamento auditivo é entendido como o processamento das informações acústicas, que analisa e interpreta os sinais acústicos. A fala corresponde a um desses sinais.

Quando discriminamos mudanças de ordem temporal na onda sonora, realizamos o processamento auditivo temporal. Tal processamento é fundamental para a fala (estímulos verbais) e para a música (estímulos não verbais), pois a ordem temporal acústica dos elementos é necessária para a compreensão da informação contida na mensagem ${ }^{(1)}$.

O processamento temporal é dividido em quatro habilidades auditivas: ordenação ou sequencialização temporal, integração ou somação temporal, mascaramento temporal, e resolução ou discriminação temporal ${ }^{(1-3)}$. A habilidade auditiva de resolução temporal é o tempo mínimo necessário para segmentar os eventos acústicos. Essa habilidade pode ser verificada por meio do teste de detecção de gap, cujo objetivo é fazer com que sejam percebidos esses intervalos de silêncio durante a emissão do estímulo $^{(4)}$. Na literatura é possível observar que crianças com dificuldades de aprendizagem e de leitura apresentam alterações nessa habilidade ${ }^{(5)}$.

O teste Gaps-in-Noise (GIN) ${ }^{(1)}$ avalia a habilidade de resolução temporal utilizando intervalos de silêncio, os quais estão inseridos em trechos de ruído branco apresentados de maneira binaural. Para que o teste $\mathrm{GIN}^{(1)}$ faça parte da bateria de testes do processamento auditivo, são necessários critérios de normalidade para ouvintes normais. No Brasil, esses critérios foram realizados em adultos sem problemas auditi$\operatorname{vos}^{(4)}$ e em crianças de 11 e 12 anos de idade sem problemas auditivos $^{(6)}$.

Estudo realizado com crianças de 11 e 12 anos de idade obteve a média dos limiares de detecção de gap de 5,05 ms em ambas as orelhas. Além disso, não houve diferença no desempenho do teste GIN de acordo com faixa etária, gênero e orelha avaliada ${ }^{(7)}$.

A relevância desse trabalho está no fato de se obter os critérios de normalidade do teste GIN para crianças de 9 anos de idade, que ainda não foram estudados na população brasileira.

Desta forma, os objetivos do estudo foram estabelecer critérios de normalidade para o teste GIN em crianças de 9 anos de idade, normo-ouvintes, obter as médias dos limiares de detecção de gap, além de verificar a influência das variáveis gênero e orelha.

\section{MÉTODOS}

Os responsáveis assinaram o Termo Consentimento Livre e Esclarecido (TCLE). O projeto foi aprovado pela Comissão de Ética para Análise de Projetos de Pesquisa (CAPPesp) da Faculdade de Medicina da Universidade de São Paulo (n $1126 / 05)$.

\section{Casuística}

Foram avaliados 40 indivíduos de 9 anos de idade, 20 do gênero masculino e 20 do gênero feminino, descartando possíveis influências relacionadas ao gênero. Para descartar influências relacionadas à orelha testada, os indivíduos foram divididos da seguinte forma:

- Grupo 1-50\% iniciou o teste GIN pela orelha direita (dez indivíduos do gênero feminino - GF1, e dez do masculino - GM1);

- Grupo 2 - 50\% iniciou o teste GIN pela orelha esquerda (dez indivíduos do gênero feminino - GF2, e dez do masculino - GM2).

\section{Materiais e procedimentos}

Para participar do estudo foram adotados os seguintes critérios de inclusão: presença de limiares audiométricos normais e desempenho no teste dicótico de dígitos maior ou igual a $95 \%$ em ambas as orelhas ${ }^{(8)}$. Foram excluídas do estudo as crianças que, de acordo com os dados da anamnese, poderiam apresentar doenças otológicas, neurológicas, cognitivas e/ou dificuldades de aprendizagem.

Os procedimentos realizados foram: anamnese (levantamento do histórico auditivo da criança); avaliação audiológica básica - audiometria tonal, logoaudiometria com os testes Limiar de Recepção de Fala (LRF) e Índice Percentual de Reconhecimento de Fala (IPRF) e imitanciometria (timpanometria e pesquisas de reflexos acústicos); e testes Dicótico de Dígitos $^{(9)}$ e Gaps-in-Noise (GIN) $)^{(1)}$, que fazem parte da bateria de avaliação do Processamento Auditivo.

O teste Dicótico de Dígitos foi utilizado como uma triagem do processamento auditivo. Todos os indivíduos deveriam apresentar desempenho dentro da normalidade para serem incluídos no estudo.

O teste GIN foi desenvolvido por Musiek em $2003^{(10)} \mathrm{e}$ padronizado para a população brasileira adulta em 2008 por Samelli e Schochat ${ }^{(11)}$.

O objetivo do referido teste é determinar o limiar de detecção de gap (intervalo de silêncio). Inseridos no estímulo de white noise (ruído branco), existem diversos gaps em posições diferentes e de durações variáveis. Os gaps poderiam ser de 2 , $3,4,5,6,8,10,12,15$ e $20 \mathrm{~ms}$. O teste é composto por uma faixa-treino e quatro faixas-teste. Cada faixa-teste consiste de diversos estímulos de seis segundos de white noise (ruído branco), com cinco segundos de intervalo entre os estímulos. Em alguns estímulos, poderia haver um único gap, dois gaps ou ainda três gaps, lembrando que metade das faixas-teste foi aplicada em uma orelha e a outra metade na orelha oposta em cada grupo (GF1; GM1; GF2; GM2), no intuito de verificar a influência dos resultados de forma igual em ambas.

A análise estatística foi realizada por meio de métodos descritivos e não-paramétricos. A estatística descritiva teve o objetivo de representar a amostra quanto às variáveis gênero e orelha. Os métodos não-paramétricos foram utilizados pois as medidas do teste GIN não apresentaram distribuição normal. Sendo assim, o teste de Mann-Whitney foi utilizado para avaliar a diferença das medidas do teste GIN entre dois grupos, e o teste dos postos sinalizados de Wilcoxon foi utilizado para a comparação da variação absoluta entre as orelhas. O nível de significância utilizado foi 0,05 (5\%), com construção de intervalos de confiança de $95 \%$. 


\section{RESULTADOS}

Os resultados foram apresentados em forma de médias de limiares de detecção de gap (Tabelas 1 e 2) e analisados de acordo com as variáveis referentes a orelha e gênero.

O objetivo da comparação entre as orelhas foi verificar se havia vantagem de uma orelha sobre a outra dentro de cada gênero. Em cada grupo foram aplicadas duas faixas em uma orelha e as outras duas na orelha oposta.

Os resultados mostraram que não houve diferença em relação ao desempenho entre as orelhas (Tabela 1). O limiar médio da orelha direita foi de $4,38 \mathrm{~ms}$ e da orelha esquerda foi de 4,24 ms.

Os resultados apontaram, ainda, que não houve diferença na comparação entre os gêneros, para nenhuma das orelhas (Tabela 2). Os resultados evidenciaram, portanto, que não há influência do gênero no desempenho dos indivíduos na habilidade de resolução temporal avaliada pelo teste GIN quanto à média de limiar de detecção de gap.

\section{DISCUSSÃO}

O processamento auditivo temporal está intimamente relacionado à percepção dos traços suprassegmentais da fala, uma vez que envolve as habilidades em perceber e armazenar estímulos acústicos. Um déficit no processamento desta habilidade auditiva pode causar baixo desempenho na habilidade de leitura e aprendizagem ${ }^{(3)}$. Este dado foi confirmado no presente estudo, que avaliou crianças com desenvolvimento dentro do esperado, sem comprometimentos na recepção e emissão da fala e da escrita.

Um estudo mostrou que crianças de 6 a 9 anos com dificuldades de aprendizagem e dislexia apresentaram habilidade de resolução temporal anormal (limiares de detecção de gap elevados), quando comparadas a um grupo controle ${ }^{(5)}$. Desta forma, recursos que avaliem a resolução temporal são fundamentais para bateria de testes da avaliação do processamento auditivo.

O teste GIN foi desenvolvido no intuito de investigar a resolução temporal, determinando o limiar de detecção de gap, ou seja, o menor intervalo de tempo onde há uma interrupção do estímulo sonoro ${ }^{(1)}$. Um estudo mostra que a sensibilidade do teste GIN está em $73 \%$ e a especificidade em $84 \%$, na identificação de pacientes com lesão do sistema auditivo central ${ }^{(1)}$. Portanto, o teste GIN pode ser considerado uma ferramenta importante para diagnosticar déficits na habilidade de resolução temporal tanto na população adulta quanto na população pediátrica ${ }^{(12)}$. Diante disso, o presente estudo tem grande importância, pois mostra os resultados do teste GIN em crianças com 9 anos, permitindo a identificação de problemas na habilidade de resolução temporal, e possibilitando a intervenção precoce.

Várias pesquisas já foram realizadas utilizando o teste GIN para avaliar o limiar de detecção de gap, em diferentes populações, principalmente adultos, com audição dentro da normalidade. Foi elaborado um quadro comparativo entre tais estudos (Quadro 1).

Observando os resultados encontrados no presente estudo foi possível verificar que na comparação entre as orelhas (direita e esquerda), tanto para o gênero masculino quanto para o feminino, não houve vantagem de uma sobre a outra. Os mesmos resultados foram encontrados em estudos anteriores, tanto para a população pediátrica quanto para a adulta( ${ }^{(1,4,6,11,12,14)}$.

A ausência de diferença entre as orelhas sugere que a resolução temporal, da forma como é avaliada no teste GIN, é um processo auditivo que se desenvolve relativamente cedo e simetricamente. Isto também sugere que a maturação do sistema auditivo procede de forma similar, tanto para a direita como para esquerda em relação à habilidade de resolução temporal ${ }^{(12)}$.

Além disso, um aspecto importante é que o teste GIN foi aplicado de forma monótica e nos testes monóticos as vias ipsi e contralaterais são ativadas. Isso resulta em desempenho semelhante das orelhas direita e esquerda ${ }^{(1)}$, confirmado assim os resultados encontrados neste trabalho, valores semelhantes para as duas orelhas.

Apesar de poucos estudos utilizando o teste GIN com a população pediátrica, acredita-se que a habilidade de resolução temporal não apresenta diferenças significativas em relação à idade, pelo menos a partir dos $7 \operatorname{anos}^{(6,12)}$. Pesquisas mostraram que aos 7 anos de idade os limiares apresentados por crianças americanas já apresentam valores próximos aos obtidos por $\operatorname{adultos}^{(12)}$

Tabela 1. Análise descritiva dos limiares obtidos nas orelhas direita e esquerda

\begin{tabular}{lccccccc}
\hline Orelha & Média & DP & Mediana & Mínimo & Máximo & IC 95\% & Valor de $p$ \\
\hline OD & 4,38 & 0,933 & 6,00 & 2,00 & 6,00 & $4,17-4,58$ & 0,21 \\
OE & 4,24 & 1,058 & 6,00 & 2,00 & 6,00 & $4,00-4,47$ & \\
\hline
\end{tabular}

Legenda: $\mathrm{OD}=$ orelha direita; $\mathrm{OE}=$ orelha esquerda; $\mathrm{DP}=$ desvio-padrão

Tabela 2. Análise descritiva dos limiares obtidos nas orelhas direita e esquerda de acordo com o gênero

\begin{tabular}{lcccccccc}
\hline Orelha & Gênero & Média & DP & Mediana & Mínimo & Máximo & $Z$ & Valor de $p$ \\
\hline \multirow{2}{*}{ OD } & $\mathrm{F}$ & 4,58 & 0,844 & 4,00 & 3,00 & 6,00 & $-1,65$ \\
& $\mathrm{M}$ & 4,18 & 0,984 & 5,00 & 2,00 & 5,00 & 0,09 \\
\multirow{2}{*}{ OE } & $\mathrm{F}$ & 4,40 & 0,928 & 4,00 & 2,00 & 6,00 & $-1,23$ & 0,21 \\
& $\mathrm{M}$ & 4,07 & 1,163 & 5,00 & 2,00 & 6,00 & \multirow{2}{*}{0} \\
\hline
\end{tabular}

Legenda: $\mathrm{OD}$ = orelha direita; $\mathrm{OE}$ = orelha esquerda; $\mathrm{F}$ = feminino; $\mathrm{M}=$ masculino; $\mathrm{DP}$ = desvio-padrão 
Quadro 1. Comparação de resultados obtidos em estudos realizados com o teste GIN

\begin{tabular}{|c|c|c|c|c|}
\hline Autor & Ano & $\begin{array}{c}\text { População } \\
\text { (anos) }\end{array}$ & Medida do limiar & Limiares de gap \\
\hline Perez $^{(6)}$ & 2009 & 11 e 12 & 4 respostas de 6 apresentações & $\begin{array}{c}5,0 \mathrm{~ms}(\mathrm{OD}) \\
5,11 \mathrm{~ms}(\mathrm{OE})\end{array}$ \\
\hline Shinn, Chermak e Musiek ${ }^{(12)}$ & 2009 & 9 & 4 respostas de 6 apresentações & $\begin{array}{c}4,6 \mathrm{~ms}(\mathrm{OD}) \\
5,10 \mathrm{~ms}(\mathrm{OE})\end{array}$ \\
\hline Musiek et al. ${ }^{(1)}$ & 2005 & 13 a 46 & 4 respostas de 6 apresentações & $\begin{array}{l}\text { 4,8 ms (OD) } \\
4,9 \mathrm{~ms}(\mathrm{OE})\end{array}$ \\
\hline Chermak, Lee ${ }^{(13)}$ & 2005 & 7 a 11 & 4 respostas de 6 apresentações & $\begin{array}{l}\text { 4,6 ms (OD) } \\
4,9 \mathrm{~ms}(\mathrm{OE})\end{array}$ \\
\hline Samelli(4) & 2005 & 18 a 31 & 3 respostas de 6 apresentações & $3,98 \mathrm{~ms}$ \\
\hline Samelli e Schochat ${ }^{(11)}$ & 2008 & 18 a 31 & 4 respostas de 6 apresentações & $4,19 \mathrm{~ms}$ \\
\hline Zaidan et al. ${ }^{(14)}$ & 2008 & 18 a 29 & 4 respostas de 6 apresentações & $\begin{array}{l}5,38 \mathrm{~ms}(\mathrm{OD}) \\
4,88 \mathrm{~ms}(\mathrm{OE})\end{array}$ \\
\hline Presente trabalho & 2009 & 9 & 4 respostas de 6 apresentações & $\begin{array}{l}4,4 \mathrm{~ms}(\mathrm{OD}) \\
4,2 \mathrm{~ms}(\mathrm{OE})\end{array}$ \\
\hline
\end{tabular}

Quando comparamos os resultados do presente estudo com os dados obtidos em outro estudo brasileiro realizado com crianças de 11 e $12 \operatorname{anos}^{(7)}$, notamos uma diferença sutil entre as médias dos limiares. Tal diferença pode ser justificada pela maturação do sistema nervoso auditivo. Segundo estudo realizado em 2005, a idade de 9 anos é uma idade considerada transitória no que diz respeito à maturação das habilidades auditivas. Por esse motivo, pode influenciar e causar uma diferença, ainda que discreta, entre os limiares dos indivíduos dessas duas faixas etárias $^{(15)}$. Cabe ressaltar que ainda não existem estudos com o teste GIN em outras faixas etárias para que possamos comparar nossos achados.

Com a normatização do teste GIN para crianças com 9 anos, o diagnóstico do Transtorno do Processamento Auditivo ganha uma ferramenta importante e necessária. Sugere-se, portanto, que os valores de limiares obtidos neste estudo (Tabela 1) sejam usados como critérios de normalidade para a idade estudada, e que limiares de detecção de gap superiores a esta média, somados a um desvio padrão, sejam considerados alterados para crianças de 9 anos de idade.

\section{CONCLUSÃO}

Neste estudo observamos que para crianças de 9 anos de idade a média dos limiares de gap é de 4,4 ms para orelha direita e 4,2 ms para orelha esquerda no teste GIN. Estes valores, acrescidos de um desvio padrão, podem ser usados como critério de normalidade para a idade. O teste GIN pode ser aplicado utilizando o mesmo critério de normalidade tanto para o gênero feminino quanto para o masculino. Além disso, também pode ser aplicado de forma binaural sem que os dados sofram influências.

\section{AGRADECIMENTOS}

Agradecemos à Fundação de Amparo à Pesquisa do Estado de São Paulo (FAPESP), pelo apoio concedido para a realização desta pesquisa, sob o processo número 08/54587- 4.

\section{REFERÊNCIAS}

1. Musiek FE, Shinn JB, Jirsa R, Bamiou DE, Baran JA, Zaidan E. GIN (Gaps-In-Noise) test performance in subjects with confirmed central auditory nervous system involvement. Ear Hear. 2005;26(6):608-18.

2. Shinn JB. Temporal processing: the basics. Hear J. 2003;56(7):52.

3. Muniz LF, Roazzi A, Schochat E, Teixeira CF, Lucena JA. Avaliação da habilidade de resolução temporal, com uso do tom puro, em crianças com e sem desvio fonológico. Rev CEFAC. 2007;9(4):550-562.

4. Samelli AG. O teste GIN (Gap in Noise): limiares de detecção de gap em adultos com audição normal [tese]. São Paulo: Universidade de São Paulo; 2005.

5. Hautus M, Setchell G, Waldie KE, Kirk IJ. Age-related improvements in auditory temporal resolution in reading-impaired children. Dyslexia. 2003;9(1):37-45.

6. Perez AP. Estudo dos limiares de detecção de gap, com o uso do teste GIN, em crianças de 11 e 12 anos [dissertação]. São Paulo: Universidade Federal de São Paulo; 2009.

7. Perez AP, Pereira LD. O teste gap in noise em crianças de 11 e 12 anos. Pró-Fono. 2010;22(1):7-12.

8. Pereira LD, Schochat E. Testes auditivos comportamentais para avaliação do processamento auditivo central. Barueri: Pró-Fono; 2011.

9. Musiek FE. Assessment of central auditory dysfunction: the dichotic digit test revisited. Ear Hear. 1983;4(2):79-83.

10. Musiek FE, Zaidan E, Baran JA, Shinn JB, Jirsa RE. Assessing temporal processes in adults with LD: the GIN test. In: Convention of American Academy of Audiology; 2004; March - April; Salt Lake City, USA. Annals. p. 203.

11. Samelli AG, Schochat E. The gaps-in-noise test: gap detection thresholds in normal-hearing young adults. Int J Audiol. 2008;47(5):238-45.

12. Shinn JB, Chermak GD, Musiek FE. GIN (Gap-In-Noise) Performance in the pediatric population. J Am Acad Audiol. 2009;20(4):229-38.

13. Chermak GD, Lee J. Comparison of children's performance on four tests of temporal resolution. J Am Acad Audiol. 2005;16(8):554-63.

14. Zaidan E, Garcia AP, Tedesco ML, Baran JA. Desempenho de adultos jovens normais em dois testes de resolução temporal. Pró-Fono. 2008;20(1):19-24.

15. Neves IF, Schochat E. Maturação do processamento auditivo em crianças com e sem dificuldades escolares. Pró-Fono. 2005;17(3):311-20. 